\title{
12 The invisibility of bisexual and pansexual bodies
}

\author{
Sexuality, appearance norms, and \\ visual identities
}

Nikki Hayfield

\section{The erasure of bisexuality and pansexuality}

The invisibility and erasure of bisexuality within the wider culture has been of central concern among activists and academics (Monro, 2015). The lack of cultural recognition of bisexuality has often been attributed to the persistence of binary understandings of (sex and) sexuality. In these binary understandings of sexuality, heterosexuality and "homosexuality" are understood to be the only possibilities. Therefore, bisexuality is overlooked - or if recognised is rapidly dismissed (e.g. Hayfield et al., 2013; Monro, 2015). In more recent years, research has indicated that pansexual people may also find that their identities are invisible in similar ways to bisexual people (King, 2013; Lapointe, 2017). Personal and social identities have often been associated with particular appearance norms, which may play a part in our wider social and cultural visibility. How we dress and appear holds the potential for the expression and recognition of our identities. This recognition serves as a form of visibility, which historically has aided in creating communities and advancing LGBTQ+ rights (Cole, 2000; Hayfield, 2013; Hayfield \& Wood, 2018; Huxley, Clarke, \& Halliwell, 2014). To date, research on visual identities and the expression and recognition of sexuality through dress and appearance has focused mainly on lesbian and gay people. There is minimal research on bisexuality and pansexuality, and seemingly none on asexual spectrum identities that relate to attraction to more than one gender, such as biromantic and panromantic. This chapter synthesises the small body of extant literature on the expression and recognition of bisexual and pansexual appearance and visual identities. In order to situate bisexual and pansexual identities within their wider context, it is necessary to also discuss lesbian and gay appearance and visual identities. 


\section{The expression and recognition of shared sexuality through visual identities}

\section{Lesbian and gay visual identities}

Dress, appearance, and style are malleable, and historically have been meaningful in the expression and recognition of identity. Those who occupy subcultural identities, including lesbian and gay sexualities, have a rich history of shared appearance norms (Clarke \& Smith, 2015; Hayfield, 2013; Hayfield $\&$ Wood, 2018; Huxley et al., 2014). These appearance norms have enabled lesbian and gay people to recognise each other - without necessarily being recognised by heterosexual people - which has aided in solidarity and the creation of safe spaces and communities. This was especially useful when same-sex acts were criminalised and lesbian and gay identities were highly oppressed. Appearance could indicate who belonged within these communities and enabled demarcated boundaries between the subcultural group and the mainstream culture (Clarke \& Smith, 2015; Clarke \& Turner, 2007; Hutson, 2010; Huxley et al., 2014).

In keeping with the early sexologists' inversion theories of gender and sexuality, gay men traditionally have been understood as feminine (or effeminate) and lesbians as masculine (Clarke \& Smith, 2015; Cole, 2000; Hayfield, 2013). Accordingly, gay men have sometimes been associated with rejecting (particular versions of) masculinity and investing in appearance practices more traditionally associated with (heterosexual) femininity. These have included neatly styled hair, jewellery and piercings, and tight-fitting fashionable clothing (e.g. Clarke \& Smith, 2015; Hayfield, 2013; Hutson, 2010). Lesbians have sometimes been associated with a more traditionally masculine appearance through clothing, short hair, sensible shoes, and the rejection of beauty practices (e.g. Clarke \& Turner, 2007; Hayfield, 2013; Huxley et al., 2014). However, such images have been problematised, and gay and lesbian looks and looking can be subtle, nuanced, and diverse. Our appearance is also likely to vary according to how different aspects of our identities intersect (e.g. our gender, sexuality, race and ethnicity, social class, and so on) (Clarke \& Spence, 2013; Hayfield \& Wood, 2018; Hutson, 2010; Huxley et al., 2014).

While lesbian and gay appearance norms have served as liberating, they have also been reported to be restrictive. Therefore, some may conform to appearance norms to a certain extent while simultaneously attempting to avoid strict adherence to what might be understood as stereotypes. Individuality is understood to be important and may have to be carefully negotiated within the dictates of "looking the part" (by dressing in ways that fit with lesbian and gay appearance norms) and "looking good" - both of which can become policed as a requirement of belonging within particular 
communities (Clarke \& Smith, 2015). In keeping with postmodern conceptualisations of identity, lesbian and gay identities have become more fragmented than in the past, and looks have diversified further. This, alongside the assimilation of lesbian and gay identities into mainstream heterosexual culture, means that these appearance norms may not be as dominant as they once were (Clarke \& Smith, 2015; Clarke \& Spence, 2013; Hayfield \& Wood, 2018; Huxley et al., 2014; Hutson, 2010).

\section{A lack of bisexual and pansexual visual identities}

Since the late 1990s, researchers have explored whether appearance norms might exist specifically for bisexual people and whether bisexuality might therefore be expressed and recognised through visual identities. The vast majority of this research has been conducted with women. Bisexual men's appearance remains under-researched or under-theorised. Findings have consistently indicated that there is no distinctive bisexual look through which people are able to express or recognise bisexuality (e.g. Clarke \& Spence, 2013; Clarke \& Turner, 2007; Daly, King, \& Yeadon-Lee, 2018; Hartman, 2013; Hayfield et al., 2013; Huxley et al., 2014; Taub, 1999). Nonetheless, appearance is important to some bisexual people in relation to their identities (although perhaps less so than it is to lesbian and gay people; see Clarke \& Spence, 2013; Clarke \& Turner, 2007). Research has identified that bisexual people recognise lesbian and gay appearance norms and that some consider how they might negotiate their appearance to express their bisexuality (e.g. Clarke \& Spence, 2013; Daly et al., 2018; Hartman, 2013; Hayfield et al., 2013; Huxley et al., 2014; Taub, 1999).

In the main, there are three ways in which bisexual women in particular have constructed their appearance and visual identities. The first of these is through "borrowing" aspects of lesbian and gay dress and appearance. The second is through adopting looks that are somewhat androgynous, by blending elements of masculinity (broadly associated with lesbian styles) and femininity (broadly associated with heterosexual appearance) in how they look. This perhaps reflects early sexologists' conceptualisations of bisexuality as a mixture of masculinity and femininity, and mirrors the notion of bisexuality as "somewhere in between" heterosexual and lesbian and gay identities. It may also follow from the androgynous looks common within some feminist communities, of which bisexual women were often a part. Third, some bisexual women have reported embracing appearances that are funky, flamboyant, or associated with alternative looks and looking (e.g., hippie, Goth, punk, and so on) including through piercings and tattoos (Clarke \& Spence, 2013; Daly et al., 2018; Hartman, 2013; Hayfield et al., 2013; Huxley et al., 2014; Taub, 1999). More broadly, bisexual participants have sometimes discussed how their bisexuality and the lack of a specific bisexual look offer them the freedom to express individuality, be playful with their appearance, and present an authentic version of themselves, rather 
than having to adhere to any rigid appearance norms (Clarke \& Spence, 2013; Hartman, 2013; Hayfield et al., 2013). Finally, the gender of a current partner or partners may factor in how bisexual women dress and appear (Davila et al., 2019; Hayfield et al., 2013; Taub, 1999). Bisexual women may thus negotiate their visual identities by fitting with lesbian aesthetics, by blending masculine and feminine looks and appearing androgynously, or by looking alternative. However, none of these is distinctive to bisexuality per se, and therefore these options seem unlikely to make bisexual people recognisable. If bisexual people cannot be read as bisexual, then they cannot be visible to others through dress and appearance; instead, the only way to convey bisexuality may be through direct verbal statements (Hayfield et al., 2013; Maliepaard, 2020). This lack of a discernable bisexual visual identity feasibly plays a part in the broader cultural invisibility of bisexuality.

To date, there has seemingly been no research that has specifically focused on pansexual appearance and visual identities. However, in research focused on other topics, participants have made reference to visibility and dress and appearance. A recent study (Davila et al., 2019) focused on whether or how bisexual (70.7 per cent), pansexual (12.9 per cent), and queer (11.1 per cent) participants tried to make their sexuality visible to others. Over half (57.6 per cent) reported that they did try to make themselves visible. One way in which they did so was through visual displays, including changing their dress to be "more or less masculine and feminine" or wearing "genderneutral clothing" (Davila et al., 2019, p. 205). Some participants also reported wearing clothing or jewellery in Pride (e.g. rainbow) or bisexual (e.g. pink, blue, and purple) colours, and having tattoos (Davila et al., 2019). It is not possible from this report to ascertain whether those who engaged in these strategies included any of the pansexual participants. Therefore, it is difficult to know whether this study replicates previous findings in relation to bisexual appearance or extends them to indicate that pansexual people may engage in similar practices.

\section{The intersections of identities}

As for all sexualities, those who are pansexual and bisexual are likely to negotiate multiple and intersecting identities. In a study focused on bisexual and pansexual people's faith and sexuality, one pansexual participant mentioned clothing. Alex (Mexican American) grew up within a cultural context where the expectation was that women should wear dresses, and her mother had requested that she modify her appearance by removing her baseball cap, because women should "look a certain way" (Levy \& Harr, 2018, p. 199). Pansexual and bisexual students have reported feeling that both their multiracial and their bisexual and pansexual identities were invisible to others. Therefore, they were at risk of their sexual and racial identities being misread or ignored. Some engaged in practices to try to make their identities visible to others, including through clothes, tattoos, and other 
symbols - which they recognised as an aspect of fitting in and belonging within their identity groups (e.g. King, 2013).

Pansexuality has sometimes been construed as an identity that is explicitly inclusive of trans people, and those who identify as pansexual and bisexual may also identify as trans and/or with identities that disrupt gender binaries (e.g. Gonel, 2013; Morandini, Blaszczynski, \& Dar-Nimrod, 2017). Historically, gender has been understood within a fixed and binary model. These dichotomous understandings of gender resulted in the notion that trans people were born (and "trapped") in "the wrong body". Therefore, the assumption was that to be trans inevitably meant a desire to transition and become "opposite" to their gender assigned at birth, often through hormonal and sometimes surgical interventions. However, understandings of trans identities have diversified. Increasing numbers of people do not seek medical changes to their gender. Further, many identify with terms that capture fluidity and disrupt the binaries of male/female, masculine/feminine, and man/woman (e.g. agender, genderfluid, genderqueer, non-binary, and pangender) (Diamond \& Butterworth, 2008; Garrison, 2018; Richards et al., 2016). Trans identities in general have both diversified and become increasingly culturally visible, which has resulted in trans people's (identities and) appearance coming under constant scrutiny (e.g. Garrison, 2018).

Individual presentations of our gender are impossible to opt out of. Those who are trans may manage their appearance in relation to their gender through their dress, demeanour, and wider appearance practices (Diamond \& Butterworth, 2008; Garrison, 2018; Richards et al., 2016). For example, some genderqueer bisexual participants have reported that their appearance is "decidedly masculine" (e.g. Lori, participant in Diamond $\&$ Butterworth, 2008, p. 368). In one study, some genderqueer participants assigned female at birth reported that they managed their appearance by breast binding, growing their body hair, and having haircuts traditionally associated with men. Genderqueer participants assigned male at birth reported wearing jewellery and cosmetics, having long hair, removing body and facial hair, and altering their gait. While it may be difficult to manage appearance without drawing on conventional gender binaries, some have tried to blur the boundaries by dressing androgynously or combining aspects of dress and clothing traditionally associated with men/masculinities and with women/femininities (Rankin \& Beemyn, 2012). In so doing, those who are genderqueer/genderfluid/non-binary may be presenting their gender ambiguously and destabilising dichotomous constructions of gender (Garrison, 2018; Richards et al., 2016). Those who present as ambiguous or androgynous may find that others notice and question their gender presentation. However, the result might not be that they are read as non-binary; instead, their gender may be misrecognised by others who only draw on gender binaries in their interpretations of appearance (see Garrison, 2018).

The appearance of those who broadly identify as trans, and as bisexual or pansexual, may be particularly complex and nuanced. The idea of blending 
aspects of masculine and feminine looks and looking resonates with the literature on bisexual and (perhaps) pansexual dress and appearance. Blurring the boundaries of binaries fits with fluid understandings of both gender and sexuality, and is reflected in the wide range of contemporary identities often taken up by younger people in particular. It may be that, for trans people, negotiating appearance in relation to gender is more salient than expressing their sexuality through how they dress and appear. Nonetheless, there are likely overlaps between disrupting and blurring the binaries of gender and of sexuality - not least because these have often been understood to be so closely interrelated. Sexuality does not necessarily hold "master status", and multiple identities need to be considered and viewed through a lens of intersectionality (e.g. how identities such as gender, sexuality, race and ethnicity, social class, age, and so on intersect with each other) (see Diamond \& Butterworth, 2008; Hutson, 2010). Multiple and overlapping identities may be difficult to express or recognise through dress and appearance.

\section{The potential for heterosexual recognition of sexuality through the body}

\section{Gaydar and the recognition of sexuality}

The term "gaydar" (a portmanteau of "gay" and "radar") has been used within lesbian and gay cultures to refer to the ability to recognise other lesbian and gay people on the basis of (sometimes subtle) visual cues (Barton, 2015). Gaydar has been considered an important form of potential communication, through which lesbian and gay people may be able to identify each other, acknowledge their shared identity, identify romantic partners, and socially interact with one another (e.g. Barton, 2015). Gaydar has also become a language that may (to some extent) be understood by those who are heterosexual. Since the 1980s, researchers have explored whether and how (lesbian, gay, and) straight people might be able to detect sexuality through visual (and vocal) cues (for reviews, see Rule, 2017; for a metaanalysis, see Tskhay \& Rule, 2013; for critical reviews, see Gelman, Mattson, \& Simpson, 2018; Miller, 2018; Vasilovsky, 2018).

These quantitative studies have tended to be based on various methods of heterosexual (and/or lesbian/gay) participants being shown photographs, videos (silent or with audio), or computer animations of people. They are then asked to identify the "targets" in these sources as "heterosexual" or "homosexual" - sometimes as the main focus of the study, or as part of a wider set of tasks (e.g. Ambady, Hallahan, \& Conner, 1999; Berger et al., 1987; Johnson et al., 2007; Shelp, 2003). These studies have tended to consider accurate identification of sexuality as an important topic on the basis of attitudes to, and prejudice and discrimination towards, lesbians and gay men (e.g. Ambady et al., 1999; Johnson et al., 2007; Tskhay \& Rule, 2013). Some researchers have concluded that participants are unable to accurately 


\section{Nikki Hayfield}

ascertain sexuality any better than by chance (e.g. Berger et al., 1987, although scholars have argued that reanalysis of data from this one early study could result in statistically significant results - see Rule, 2017; Tskhay \& Rule, 2013). Others have concluded that people are somewhat able to make (at least partially) accurate judgements of sexuality on the basis of a range of visual cues (e.g. Ambady et al., 1999; Johnson et al., 2007; Shelp, 2003; Tskhay \& Rule, 2013). Those who are lesbian or gay, perhaps unsurprisingly, may accurately assess sexuality to a greater extent than heterosexual people (e.g., Ambady et al., 1999; Berger et al., 1987; Shelp, 2003). Most commonly, participants have reportedly made their assessments on the basis of appearance (e.g. choice and fit of clothing, hairstyles, and jewellery), facial shape, facial expression including smiles, eye gaze, gestures, and body posture, shape, and motion (e.g. gait) (e.g. Berger et al., 1987; Johnson et al., 2007; Shelp, 2003; see also Rule, 2017; Tskhay \& Rule, 2013).

\section{But what about bidar or pandar?}

In some studies, gaydar is defined as an ability "to distinguish between homosexual and heterosexual people", hence the underpinning definition is binary and even when gaydar is more broadly defined as "the ability to detect, judge or perceive sexual orientation", what most studies ask participants to do is choose between two categories of heterosexual or "homosexual" (Miller, 2018, p. 191, emphases added). These narrow conceptualisations of sexuality as binary reflect how bisexuality and pansexuality have been overlooked by researchers (Gelman et al., 2018; Miller, 2018; Vasilovsky, 2018), despite increases in the numbers of people who identify with these and other diverse sexualities, which relate to attraction to more than one gender.

Indeed, the most common approach within gaydar research has been to ignore bisexuality and pansexuality. This has been the case even when "targets" and participants have completed Kinsey scales (or other selfreports of sexuality) that could have enabled researchers to move beyond the binary of straight or gay (see Miller, 2018). For example, in one study, both the "targets" (whose photos and silent video clips were assessed) and the participants (who assessed the photos and videos) were asked to rate themselves on a seven-point Kinsey-type scale in response to the statement "I have homosexual tendencies" (Ambady et al., 1999, p. 541). The authors note that while most "targets" rated themselves at the extreme ends of the scale (and were therefore categorised as heterosexual or "homosexual"), one self-rated as a 4 (e.g. in the middle of the scale). They do not state whether this "target" was excluded from the study or amalgamated with the other "targets" (e.g. categorised as heterosexual or "homosexual"). The participants being asked to assess the sexuality of the "targets" were categorised as heterosexual if they rated themselves as $1-3$, or as lesbian or gay if they rated themselves 4-7 (Ambady et al., 1999). Therefore, this study 
may feasibly have included "targets" and participants who self-identified as bisexual or with other identities, yet this was not fully taken into consideration, which could arguably have impacted the results (see Miller, 2018). In another example, participants were asked to identify their own sexuality as "gay", "straight", or "other". Those who responded "other" were omitted from the study (Shelp, 2003, p.10). Even in more recent studies, researchers often make no mention of bisexuality or pansexuality, or refer to bisexuality only briefly, sometimes to state their exclusion of bisexual participants (e.g. Cox et al., 2016; see also Miller, 2018).

To date, there are seemingly only two published studies that include bisexuality as an option for participants when assessing sexuality. Ding and Rule (2012, p. 166) investigated whether facial recognition of sexuality extends beyond lesbians and gay men to the "less socially salient category" of bisexuality. They conducted three separate studies. In the first, 60 undergraduate students were asked to view photographs on a computer of 45 gay men, 44 straight men, and 41 men who self-identified as bisexual (or who indicated that they would date men or women). All the men were Caucasian, aged between 18-30 years, with no piercings or facial hair. Participants were asked to categorize these photographs trichotomously, as gay, straight, or bisexual. Participants accurately categorized gay and straight men at a level significantly greater than by chance, but could not do so for bisexual men. In the second study, the same photos were shown to another 33 undergraduate students, who were asked to rate the sexuality of the men in the photographs on a seven-point scale from "definitely gay" through to "definitely straight". When asked to use this continuum, participants were able to distinguish between straight and gay men and between straight and bisexual men at a rate higher than chance. However, they could not distinguish between gay and bisexual men. In the third study, 40 undergraduate students viewed photos of 40 lesbian, 40 straight, and 40 self-identified bisexual women. While participants were able to distinguish between lesbian and straight women and between bisexual and straight women at a rate higher than chance, this was not the case for distinguishing between bisexual and lesbian women. The authors note that participants' inability to distinguish between bisexual and gay/lesbian photographs (despite being able to distinguish between bisexual and straight) may arise as a result of participants relying on dominant binary understandings of sexuality (straight/non-straight), even though they were given the option to categorise people as bisexual (Ding \& Rule, 2012).

In a related study, Lick, Johnson, and Rule (2015) used some of the same photographs as Ding and Rule (2012). Eighty-three undergraduate students were shown 60 grayscale photographs of straight men, gay men, straight women, lesbian women, bisexual men, and bisexual women (10 of each identity). Again, participants were shown faces of White people aged between 18 and 30 years, with no facial hair or piercings. They were shown each photograph three times and asked to categorise the people in the photographs 
as "bisexual" or "not bisexual" and to complete Likert scales of how they rated the faces from "masculine" to "feminine". Participants correctly assigned those who were not bisexual (e.g. lesbian, gay, or straight) at an above-chance level (59.94 per cent of the time). However, when assigning photographs as bisexual, accuracy levels were no greater than chance (49.58 per cent of the time). Women were more likely to be categorised as bisexual than men. Those assessed by participants as "gender atypical" on the masculinity to femininity measure were more likely to be categorised as bisexual than those assessed as "gender typical" (and this notion of bisexual people being evaluated as "gender atypical" may reflect some of the ways in which bisexual people discuss expressing their identities, as discussed above). The authors conclude that gender cues guide assessments of bisexuality and that participants' above-chance identification of non-bisexual people was only on the basis of rejecting photographs of lesbian women, gay men, and straight people, rather than correctly identifying bisexual people (Lick et al., 2015). These findings highlight the importance of including bisexuality and raise questions around how or why participants are able to separate bisexual people from straight people, but not from lesbians and gay men (Miller, 2018). Yet very few studies have explicitly included bisexual "targets" in the photographs, videos, or other data-collection tools, or included bisexual people within the groups of participants who assess others' sexuality, or even taken bisexuality into consideration. To date, it would seem that there has been no inclusion of pansexual or asexual spectrum identities. This tendency towards excluding those attracted to more than one gender as either "targets" or participants may reflect some researchers' binary understandings of sexuality and contributes to the ongoing erasure and invisibility of bisexual/biromantic and pansexual/panromantic people.

Over the last few years, scholars have critiqued gaydar research and highlighted a myriad of conceptual and methodological limitations. (For in-depth discussions of issues, including selection of stimulus photographs; decontextualized design and ecologically invalid settings; accuracy rates; effect sizes, response biases, and errors, see Cox et al., 2016; Gelman et al., 2018; Miller, 2018). Participants may be assessing sexuality on the basis of stereotypes; therefore, rather than providing evidence for the existence of gaydar, these studies may instead indicate that participants are knowledgeable about (overly simplistic) stereotypes of lesbian and gay people as gender atypical (Cox et al., 2016; Gelman et al., 2018; Miller, 2018). Indeed, a key question has been whether participants are able to identify sexuality, or whether what they are actually assessing is based on gender inversion models - which may further perpetuate their existence and therefore the exclusion of bisexuality and pansexuality (Miller, 2018; Vasilovsky, 2018). Most recently, and most controversially, one study concluded that artificial intelligence can detect lesbian and gay sexuality from facial images (taken from a dating website), which the authors concluded was on the basis of facial shape and features (Wang \& Kosinski, 2018; see Gelman et al., 2018 
and Miller, 2018 for critical discussion). While dress and appearance have been interpreted within a framework of socially and culturally produced shared meanings, in contrast, many of the findings of gaydar research are often (overly simplistically) reduced to hormonal, genetic, biological explanations of sexuality. These implicitly locate sexuality as inherent, immutable, and universal, which has political implications and may not necessarily be advantageous (see Vasilovsky, 2018).

\section{Qualitative explorations of perceptions of lesbian, gay, and bisexual appearance}

Researchers have also explored how heterosexual (and lesbian, gay, and bisexual) people perceive sexuality and appearance using qualitative methods (Hayfield, 2013; Hayfield \& Wood, 2018). In one study, 36 (mainly heterosexual) students completed a survey in which the participants were all asked how they would describe what a lesbian woman, gay man, heterosexual woman, heterosexual man, bisexual woman, and bisexual man might look like, and how they might "potentially recognise them from their appearance" (Hayfield, 2013, p. 18). Participants were able to describe effeminate gay men and masculine butch lesbians, with some also referring to femme lesbians. They were also able to describe heterosexual appearance on the basis of traditional and dichotomous notions of gender, as shorthaired muscular masculine men and long-haired slim feminine women. They often reported that they saw these images as stereotypes rather than what people really looked like. However, most participants were unable to describe any bisexual appearance. Some explicitly commented that they could not describe a bisexual look or distinguish bisexuality on the basis of appearance. The few participants who did provide descriptions of bisexuality sometimes indicated that bisexual people might look heterosexual or gay/lesbian. Others (including one heterosexual and one bisexual participant) suggested that bisexual people might embrace alternative and Goth looks (Hayfield, 2013). Nonetheless, what dominated was an inability to picture bisexual appearance in contrast to being able to describe (particular versions of) heterosexual and lesbian and gay appearance.

Most recently, Hayfield and Wood (2018) presented 54 (mainly heterosexual student) participants with a hypothetical scenario about a bisexual, lesbian, or heterosexual woman going on a date. They were randomly allocated to one version of this scenario (e.g. a bisexual or lesbian or heterosexual character) and asked to complete a story about the character's preparation for the date, how the date went, and what happened afterwards. They then produced a cartoon image (using Bitstrips) of the character in their story. Traditional notions of gender and heterosexuality dominated, and there was little diversity in how the characters were described or depicted. Most were portrayed in keeping with traditional heterosexual femininity as having long hair, removing body hair, and wearing dresses and makeup. 
Only a few participants portrayed their lesbian character in any ways that mirrored lesbian appearance norms and none of the participants assigned to the bisexual character suggested any bisexual appearance. These findings may indicate that participants had little cultural knowledge of lesbian and bisexual cultures to draw upon when writing their stories. Alternatively, the results could reflect that particular looks in relation to sexuality are somewhat diminishing, and therefore are not available for younger people to draw upon - perhaps due to the diversification and/or the assimilation of lesbian and gay identities into mainstream culture (Hayfield \& Wood, 2018). On the whole, these studies mirror research with bisexual participants in suggesting that bisexual appearance norms may be few and far between and little recognised by others.

\section{Conclusion}

To date, psychologists, sociologists, anthropologists, and others have explored the expression and recognition of lesbian, gay and (to some extent) bisexual appearance within a framework of socially and culturally produced shared meanings. It would seem that some genders and sexualities can potentially be expressed through appearance. In contrast, there are seemingly no known appearance norms or visual scripts that can easily make bisexual and pansexual (or, by extension, biromantic and panromantic) identities visible. Further, multiple and overlapping identities may be particularly challenging to communicate and there may be limited opportunities to express these identities through appearance.

Psychologists and others have also taken a variety of approaches to consider whether heterosexual (and lesbian, gay, and bisexual) participants might recognise sexuality through bodies and appearance. While lesbian and gay identities may be recognisable to some, the dominant picture is of a lack of recognition of bisexuality and pansexuality - which reflects wider bisexual and pansexual invisibility. Some scholars have suggested that the lack of recognition of bisexuality may have consequences for the mental health and wellbeing of bisexual people (see Hayfield et al., 2013; Monro, 2015). This may extend to pansexual identities. Further, lesbian and gay looks may be diminishing and be less dominant than they once were; therefore, it seems unlikely that bodies are sites through which invisibility will become addressed in the future. Nonetheless, it remains important to be aware of the ways in which bodies intersect with gender and sexuality alongside other aspects of identities - and may therefore be sites of in/validation and in/visibility.

\section{References}

Ambady, N., Hallahan, M., \& Conner, B. (1999). Accuracy of judgments of sexual orientation from thin slices of behavior. Journal of Personality and Social Psychology, 77(3), 538-47. doi: 10.1037/0022-3514.77.3.538 
Barton, B. (2015). How like perceives like: Gay people on "gaydar". Journal of Homosexuality, 62(12), 1615-1637. doi: 10.1080/00918369.2015.1091207

Berger, G., Hank, L., Rauzi, T., \& Simkins, L. (1987). Detection of sexual orientation by heterosexuals and homosexuals. Journal of Homosexuality, 13(4), 83-100. doi: 10.1300/J082v13n04_05

Clarke, V., \& Smith, M. (2015). "Not hiding, not shouting, just me": Gay men negotiate their visual identities. Journal of Homosexuality, 62(1), 4-32. doi: 10.1080/ 00918369.2014.957119

Clarke, V., \& Spence, K. (2013). "I am who I am”? Navigating norms and the importance of authenticity in lesbian and bisexual women's accounts of their appearance practices Psychology \& Sexuality, 4(1), 25-33. doi: 10.1080/ 19419899.2013.748240

Clarke, V., \& Turner, K. (2007). Clothes maketh the queer? Dress, appearance and the construction of gay, lesbian and bisexual identities. Feminism and Psychology, 17(2), 267-76. doi: 10.1177/0959353507076561

Cole, S. (2000). Don we now our gay apparel: Gay men's dress in the twentieth century. Oxford: Berg. doi: 10.2752/9781847888679

Cox, W.T., Devine, P.G., Bischmann, A.A., \& Hyde, J.S. (2016). Inferences about sexual orientation: The roles of stereotypes, faces, and the gaydar myth. The Journal of Sex Research, 53(2), 157-71. doi: 10.1080/00224499.2015.1015714

Daly, S., King, N., \& Yeadon-Lee, T. (2018). "Femme it up or dress it down": Appearance and bisexual women in monogamous relationships. Journal of Bisexuality, 18(3), 257-77. doi: 10.1080/15299716.2018.1485071

Davila, J., Jabbour, J., Dyar, C., \& Feinstein, B.A. (2019). Bi+ visibility: Characteristics of those who attempt to make their bisexual+ identity visible and the strategies they use. Archives of Sexual Behavior, 48(1), 199-211. doi: 10.1007/ s10508-018-1284-6

Diamond, L.M., \& Butterworth, M. (2008). Questioning gender and sexual identity: Dynamic links over time. Sex Roles, 59(5-6), 365-76. doi: 10.1007/ s11199-008-9425-3

Ding, J.Y., \& Rule, N.O. (2012). Gay, straight, or somewhere in between: Accuracy and bias in the perception of bisexual faces. Journal of Nonverbal Behavior, 36(2), 165-76. doi: 10.1007/s10919-011-0129-y

Garrison, S. (2018). On the limits of "trans enough": Authenticating trans identity narratives. Gender \& Society, 32(5), 613-37. doi: 10.1177/0891243218780299

Gelman, A., Mattson, G., \& Simpson, D. (2018). Gaydar and the fallacy of decontextualized measurement. Sociological Science, 5, 270-80. doi: 10.15195/ v5.a12

Gonel, A.H. (2013). Pansexual identification in online communities: Employing a collaborative queer method to study pansexuality. Graduate Journal of Social Science, 10(1), 36-59.

Hartman, J.E. (2013). Creating a bisexual display: Making bisexuality visible. Journal of Bisexuality, 13(1), 39-62. doi: 10.1080/15299716.2013.755727

Hayfield, N. (2013). "Never judge a book by its cover?": Students' understandings of lesbian, gay, and bisexual appearance. Psychology \& Sexuality, 4(1), 16-24. doi: 10.1080/19419899.2013.748261

Hayfield, N., Clarke, V., Halliwell, E., \& Malson, H. (2013). Visible lesbians and invisible bisexuals: Appearance and visual identities among bisexual women. Women's Studies International Forum, 40(1), 172-82. doi: 10.1016/j.wsif.2013.07.015 
Hayfield, N., \& Wood, M. (2019). Looking heteronormatively good! Combining story completion tasks with Bitstrips to explore understandings of sexuality and appearance. Qualitative Research in Psychology, 16(1), 115-35. doi: 10.1080/ 14780887.2018.1536390

Hutson, D.J. (2010). Standing out/fitting in: Identity, appearance, and authenticity in gay and lesbian communities. Symbolic Interaction, 33(2), 213-33. doi: 10.1525/ si.2010.33.2.213

Huxley, C., Clarke, V., \& Halliwell, E. (2014). Resisting and conforming to the "lesbian look": The importance of appearance norms for lesbian and bisexual women. Journal of Community \& Applied Social Psychology, 24(3), 205-19. doi: $10.1002 /$ casp. 2161

Johnson, K.L., Gill, S., Reichman, V., \& Tassinary, L.G. (2007). Swagger, sway, and sexuality: Judging sexual orientation from body motion and morphology. Journal of Personality and Social Psychology, 93(3), 321-34. doi: 10.1037/ 0022-3514.93.3.321

King, A.R. (2013). Mixed messages: How primary agents of socialization influence adolescent females who identify as multiracial-bisexual. Journal of LGBT Youth, 10(4), 308-27. doi: 10.1080/19361653.2013.825198

Lapointe, A.A. (2017). "It's not pans, it's people": Student and teacher perspectives on bisexuality and pansexuality. Journal of Bisexuality, 17(1), 88-107. doi: 10.1080/ 15299716.2016.1196157

Levy, D.L., \& Harr, J. (2018). "I never felt like there was a place for me”: Experiences of bisexual and pansexual individuals with a Christian upbringing. Journal of Bisexuality, 18(2), 186-205. doi: 10.1080/15299716.2018.1431169

Lick, D.J., Johnson, K.L., \& Rule, N.O. (2015). Disfluent processing of nonverbal cues helps to explain anti-bisexual prejudice. Journal of Nonverbal Behavior, 39(3), 275-88. doi: 10.1007/s10919-015-0211-y

Maliepaard, E. (2020). Spaces with a bisexual appearance: re-conceptualizing bisexual space(s) through a study of bisexual practices in the Netherlands. Social \& Cultural Geography, 21(1), 45-63. doi: 10.1080/14649365.2018.1454979

Miller, A.E. (2018). Searching for gaydar: Blind spots in the study of sexual orientation perception. Psychology \& Sexuality, 9(3), 188-203. doi: 10.1080/ 19419899.2018.1468353

Monro, S. (2015). Bisexuality: Identities, politics, and theories. Basingstoke: Palgrave Macmillan. doi: 10.1057/9781137007315

Morandini, J.S., Blaszczynski, A., \& Dar-Nimrod, I. (2017). Who adopts queer and pansexual sexual identities? Journal of Sex Research, 54(7), 911-22. doi: 10.1080/ 00224499.2016 .1249332

Rankin, S., \& Beemyn, G. (2012). Beyond a binary: The lives of gender-nonconforming youth. About Campus, 17(4), 2-10. doi: 10.1002/abc.21086

Richards, C., Bouman, W.P., Seal, L., Barker, M.J., Nieder, T.O., \& T'Sjoen, G. (2016). Non-binary or genderqueer genders. International Review of Psychiatry, 28(1), 95-102. doi: 10.3109/09540261.2015.1106446

Rule, N.O. (2017). Perceptions of sexual orientation from minimal cues. Archives of Sexual Behavior, 46(1), 129-39. doi: 10.1007/s10508-016-0779-2

Shelp, S.G. (2003). Gaydar. Journal of Homosexuality, 44(1), 1-14. doi: 10.1300/ J082v44n01_01

Taub, J. (1999). Bisexual women and beauty norms: A qualitative examination. Journal of Lesbian Studies, 3(4), 27-36. doi: 10.1300/J155v03n04_04 
Tskhay, K.O., \& Rule, N.O. (2013). Accuracy in categorizing perceptually ambiguous groups: A review and meta-analysis. Personality and Social Psychology Review, 17(1), 72-86. doi: 10.1177/1088868312461308

Vasilovsky, A.T. (2018). Aesthetic as genetic: The epistemological violence of gaydar research. Theory \& Psychology, 28(3), 298-318. doi: 10.1177/ 0959354318764826

Wang, Y., \& Kosinski, M. (2018). Deep neural networks are more accurate than humans at detecting sexual orientation from facial images. Journal of Personality and Social Psychology, 114(2), 246-57. doi: 10.1037\%2Fpspa0000098 